\title{
Global dynamics of an autoparametric spring-mass-pendulum system
}

\author{
Khalid EI Rifai · George Haller • Anil K. Bajaj
}

Received: 29 September 2004 / Accepted: 21 June 2006 / Published online: 22 September 2006

(C) Springer Science + Business Media B.V. 2006

\begin{abstract}
In this paper, a study of the global dynamics of an autoparametric four degree-of-freedom (DOF) spring-mass-pendulum system with a rigid body mode is presented. Following a modal decoupling procedure, typical approximate periodic solutions are obtained for the autoparametrically coupled modes in 1:2 internal resonance. A novel technique based on forward-time solutions for finite-time Lyapunov exponent is used to establish global convergence and domains of attraction of different solutions. The results are compared to numerically constructed domains of attraction in the plane of initial position and initial velocity for the pendulum. Simulations are also provided for a few interesting cases of interest near critical values of parameters. Results also shed some light on the role played by other modes present in a multi-DOF system in shaping the overall system response.
\end{abstract}

Keywords Autoparametric pendulum - Domains of attraction · Finite-time Lyapunov exponents · Global dynamics · Internal resonance

K. E. Rifai · G. Haller

Department of Mechanical Engineering, Massachusetts Institute of Technology, Cambridge, MA 02139, USA

\section{A. K. Bajaj $(\square)$}

School of Mechanical Engineering, Purdue University, 585

Purdue Mall, West Lafayette, IN 47907-2088, USA

e-mail: bajaj@ecn.purdue.edu

\section{Introduction}

Dynamical systems possessing both flexible and rigid body modes arise in many practical applications, including robotics and hard disk drive systems. The vibrational response of harmonically excited linear massspring-damper systems exhibiting modal as well as rigid body dynamics is well understood. Due to the linearity of system dynamics, the vibrational response can be projected onto the normal mode coordinates and reduced to analyzing the response of only a flexible system or that of a rigid body system. For nonlinear systems, however, this decoupling is not possible since no general modal decoupling procedure is available. Harmonically excited spring-mass-damper systems with nonlinear components are known to undergo complicated dynamics in their vibratory motions near resonant conditions [1, 2]. More specifically, linear spring-mass-damper systems when coupled to a pendulum possess quadratic nonlinearities due to inertial coupling with the rotational motion of the pendulum even though the pendulum by itself only has geometric nonlinearities. These nonlinearities, even if assumed to be weak and usually approximated as quadratic for small excitation and response amplitudes, lead to large vibration affects with many scenarios.

The case of 1:2 internal resonance between a pendulum and another structural or flexible mode that is resonantly excited has received tremendous attention in recent years. Many researchers have studied this 
class of problems where 1:2 internal resonance leads to nonlinear modal coupling in a natural manner. See extensive literature reviews in $[1,2]$. Examples of nonlinear modal coupling due to $1: 2$ internal resonance include pitch and roll motion in ships and surface waves in fluid containing cavities. Others [3-7] have studied these systems with a view to using the pendulum as a vibration absorber or neutralizer. To enhance the effectiveness of the absorber, a computer-controlled mass sliding over a collar on an oscillating rod was used in [8] as a tunable vibration absorber, and active vibration control laws have been also developed on the basis of this concept (e.g., see [9]). One important advantage of the autoparametric vibration absorber over linear passive vibration attenuation schemes based on dynamic vibration absorbers [10] is that no additional resonances in the neighborhood of excitation are created when the secondary system is introduced into the excited system. Banarjee et al. [11] studied a system consisting of an $n$ degree-of-freedom (DOF) massspring-damper assembly with a pendulum attached to the $k$ th mass, and investigated periodic solutions and possible bifurcation by using the asymptotic method of averaging. More recently, Vyas and Bajaj [12] again considered a single spring-mass system, but now with multiple pendulums tuned at slightly different frequencies, and showed significant increase in the effective bandwidth of the autoparametric pendulum as a vibration absorber.

Almost all the above studies were primarily local in that they were limited to finding small steady-state periodic or other more complex solutions near the equilibrium position of the unforced system, and included their stability and bifurcation behavior. Only the work of Lee and Hsu [13] has investigated the domains of attraction of a two DOF spring-pendulum system for dynamics within a local region in the pendulum's plane. To study the domains of attraction of periodic solutions, the authors used the cell-to-cell mapping technique developed by Hsu [14]. The domain of attraction of an invariant set is the set of all initial conditions for whom the solutions converge to the invariant set. Thus, the domains of attraction for a system provide an understanding of the transient dynamics of the system as it evolves or asymptotically moves toward a steady state, and are known to be determined by the global stable and unstable manifolds of hyperbolic invariant sets for the system. The basin boundaries of attractors have received much attention in the context of sensitive dependence on initial conditions [15]. Even when the system does not have a chaotic attractor, it can exhibit complex transient dynamics if the basin boundaries of periodic or other regular attractors are fractal in nature. We should also note that basin boundaries are invariant manifolds along which sensitive dependence to initial conditions must hold. Indeed, initial conditions selected on different sides of the basin boundary approach different invariant sets, and hence develop a finite distance, no matter how close they were initially. The converse is certainly not true: sensitive sets are not necessarily basin boundaries.

The domains of attraction in a region of the statespace can also be constructed by direct numerical integration of the equations of motion over a grid of initial conditions. This is a very numerically intensive and inefficient approach. A more efficient approach can be based on the determination of stable and unstable manifolds or hyperbolic behavior in time-dependent velocity fields. The hyperbolic behavior is, in general, characterized by distribution of finite-time Lyapunov exponents in the flow. Haller [16] has recently derived rigorous results that allow one to locate finite-time hyperbolic sets and their local stable and unstable manifolds in time-dependent velocity fields. He also provides a numerical algorithm for computing a first approximation to the uniformly hyperbolic sets in twodimensional velocity fields. It has also been shown $[17,18]$ that contours or level curves of finite-time Lyapunov exponents exhibit striking resemblance to stable and unstable manifolds which define the basin boundaries.

In this work, we consider a system consisting of an autoparametric pendulum that is coupled to a 3-DOF linear spring-mass-damper system. The 3-DOF system possesses one rigid body mode and two elastic modes. Following the problem setup, a modal decoupling procedure is performed in Section 2 on the nondimensional form of equations to study the dynamics of the system. In Section 3, approximate steady-state periodic solutions of the system to a harmonic force excitation acting on the lowest mass are then developed by using the harmonic balance approach. The focus is on the dynamics of the system when the resonantly excited highest-frequency mode is in near 2:1 internal resonance with the pendulum linear frequency. For parameter combinations with multiple periodic solutions, the domains of attraction of coexisting periodic solutions are then considered in Section 4. The domains of 
attraction in a subset region of the state-space of the system are first obtained by direct numerical integration over a grid of initial conditions. Here the initial conditions of importance are those for the pendulum, that is, its angular position and angular velocity. Subsequently, the maximal finite-time Lyapunov exponent is computed for a grid of initial conditions in pendulum subspace, and level curves for the Lyapunov exponent are contrasted with the domains of attraction determined by direct numerical simulation. The work concludes in Section 5 with some remarks.

\section{Problem formulation}

Consider the 3-DOF linear mass-spring-damper assembly in Fig. 1, which has a pendulum attached to the second mass, and is subjected to a single frequency harmonic excitation applied to the bottom mass. The masses, springs, and the dashpots in the 3-DOF system are all identical and the system is in vertical plane. Note that the system with pendulum in downward or upward vertical position can also translate as a rigid body, which represents the first mode of the system. Clearly, the system shown may exhibit linear "lockedpendulum" vibrations (pendulum settled to the lower or upper equilibrium position) for the 4-DOF system with $\theta=0$, or nonlinear oscillations in a system in which all four DOFs are active (nontrivial pendulum oscillations in addition to vibrations of the spring-mass-damper system). Naturally, the linear forced oscillations with pendulum in upward equilibrium position are expected to be unstable, while those with the pendulum in downward equilibrium position are expected to be stable at least for some excitation parameters. The focus of this study is on the case when a 1:2 internal resonance exists between the pendulum (rotational) mode and the third locked-pendulum mode, and this third linear mode is in near-resonance with external excitation. It is assumed that the coordinates $x_{1}, x_{2}$, and $x_{3}$ are measured from the equilibrium position of the system which exists under the action of constant upward forces of magnitudes $M g,(M+m) g$, and $M g$, applied, respectively, to the three blocks. These forces, not shown in the figure, compensate for the gravitational forces on the blocks. The equations of motion governing the motion of the system are then given as

$$
M \ddot{x}_{1}+c_{1}\left(\dot{x}_{1}-\dot{x}_{2}\right)+k_{1}\left(x_{1}-x_{2}\right)=P_{0} \cos \omega t,
$$

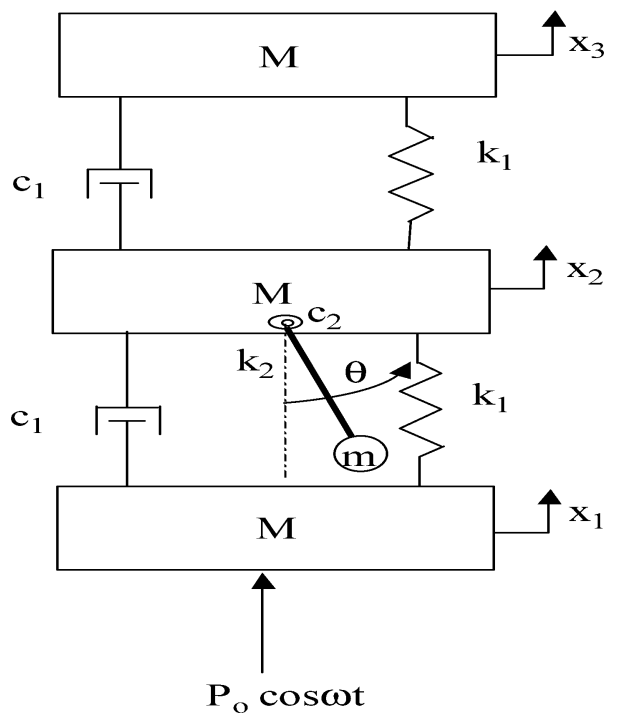

Fig. 1 The three-degree-of-freedom spring-mass-damper system with a nonlinear pendulum attached to the middle block

$$
\begin{aligned}
& (M+m) \ddot{x}_{2}+c_{1}\left(2 \ddot{x}_{2}-\dot{x}_{1}-\dot{x}_{3}\right)+k_{1}\left(2 x_{2}-x_{1}-x_{3}\right) \\
& \quad+m l\left(\ddot{\theta} \sin \theta+\dot{\theta}^{2} \cos \theta\right)=0, \\
& M \ddot{x}_{3}+c_{1}\left(\dot{x}_{3}-\dot{x}_{2}\right)+k_{1}\left(x_{3}-x_{2}\right)=0, \\
& m l^{2} \ddot{\theta}+m l\left(\ddot{x}_{2}+g\right) \sin \theta+c_{2} \dot{\theta}+k_{2} \theta=0 .
\end{aligned}
$$

It is important to note that the 4-DOF system with pendulum has $\theta=0$ as a solution. The coupling in the pendulum dynamics and the dynamics of the 3-DOF linear system arises only through terms in the equation for the second block. For small amplitude oscillations, if only linear terms in generalized coordinates and velocities are retained in the model, the three coordinates $x_{1}, x_{2}$, and $x_{3}$ are uncoupled from the linear pendulum equation. Now, the natural frequencies of the 3-DOF linear undamped system (system without pendulum) are given by $\Omega_{1}=0, \Omega_{2}=$ $\sqrt{k_{1} / M}$, and $\Omega_{3}=\sqrt{3 k_{1} / M}$. For the 4-DOF linear system that includes pendulum, it is easy to show that the second natural frequency $\Omega_{2}=\sqrt{k_{1} / M}$ remains the same, $\omega_{2 n}=\Omega_{2}$, while the third frequency $\Omega_{3}$ is modified to $\omega_{3 n}=\sqrt{k_{1} / M} \sqrt{(3 M+m) /(M+m)}=$ $\Omega_{2} \sqrt{(3+R) /(1+R)}$. The additional natural frequency is that for the pendulum with $\omega_{p}=\sqrt{\omega_{g}^{2}+\omega_{k}^{2}}$ where $\omega_{g}=\sqrt{g / l}$ and $\omega_{k}=\sqrt{k_{2} / m l^{2}}$. These two frequencies, respectively, represent the linear gravity and stiffness controlled natural frequencies of the pendulum about the bottom equilibrium position. In 
the remaining work, we will only consider pendulum motions near $\theta=0$, that is, motions in which the pendulum is near its bottom equilibrium position. The complete (4-DOF) system then has four linear natural modes: three of them are the "locked-pendulum" versions of the modes of the 3-DOF linear system, and the fourth one is the additional pendulum mode of vibration. We now define the following non-dimensional parameters:

$$
\begin{aligned}
\tau & =\omega t, \quad \eta_{i}=x_{i} / l, \quad R=m / M, \quad \varepsilon=P_{0} / k_{1} l \\
\zeta_{1} & =c_{1} / 2 M \Omega_{2}, \quad \zeta_{2}=c_{2} / 2 m l^{2} \sqrt{\omega_{g}^{2}+\omega_{k}^{2}} \\
\alpha & =\omega / \Omega_{2}, \quad \beta_{c}=\sqrt{\beta_{2}^{2}+\beta_{2}^{2}} \\
\beta_{1} & =\omega_{g} / \omega_{2 n}, \quad \beta_{2}=\omega_{k} / \omega_{2 n} .
\end{aligned}
$$

With these parameters, Equations (1)-(3) reduce to the following non-dimensional form:

$$
[M]\{\eta\}^{\prime \prime}+[C]\{\eta\}^{\prime}+[K]\{\eta\}=\{F\},
$$

where

$$
\begin{aligned}
& {[M]=\left[\begin{array}{ccc}
\frac{1}{1+R} & 0 & 0 \\
0 & 1 & 0 \\
0 & 0 & \frac{1}{1+R}
\end{array}\right],} \\
& {[C]=2 \hat{\zeta}_{1} \hat{\alpha}\left[\begin{array}{ccc}
1 & -1 & 0 \\
-1 & 2 & -1 \\
0 & -1 & 1
\end{array}\right],} \\
& {[K]=\hat{\alpha}^{2}\left[\begin{array}{ccc}
1 & -1 & 0 \\
-1 & 2 & -1 \\
0 & -1 & 1
\end{array}\right],} \\
& \varepsilon \hat{\alpha}^{2} \cos \tau \\
& \{F\}=\left\{\begin{array}{c}
-\gamma\left(\theta^{\prime \prime} \sin \theta+\theta^{\prime 2} \cos \theta\right) \\
0
\end{array},\{\eta\}=\left\{\begin{array}{l}
\eta_{1} \\
\eta_{2} \\
\eta_{3}
\end{array}\right\},\right.
\end{aligned}
$$

and $\gamma=R /(R+1), \quad \hat{\zeta}_{1}=\zeta_{1} / \sqrt{1+R}, \quad \hat{\alpha}=1 /$ $(\alpha \sqrt{1+R})$. Note that there is also the pendulum equation for the 4-DOF system. The influence of the pendulum on block motions is reflected in the vector $F$ on the right-hand side of Equations (6).
Since the analysis here is primarily concerned with the response of the system when the third translational mode of the system is resonantly excited $\left(\omega_{3 n} / \omega \approx 1\right)$, it is natural to perform a modal transformation to uncouple the modes of the translational system. It is noteworthy that the damping and stiffness matrices are proportional. This means that the damped linear 3-DOF system can be uncoupled on the basis of the undamped modal matrix for the 3-DOF system. Let $[P]$ be the modal matrix of the undamped system (6) corresponding to the translational coordinates $\eta_{i}$ 's with $\{F\}=0$. Then, the transformation $\{\eta\}=[P]\{y\}$ transforms the system (6) into the following form:

$$
\begin{gathered}
{[P]^{\mathrm{T}}[M][P]\{y\}^{\prime \prime}+[P]^{\mathrm{T}}[C][P]\{y\}^{\prime}} \\
+[P]^{\mathrm{T}}[K][P]\{y\}=[P]^{\mathrm{T}}\{F\} .
\end{gathered}
$$

Here the components of the vector $\{y\}$, that is, $y_{1}, y_{2}$, and $y_{3}$, are the amplitudes of the three linear lockedpendulum modes of vibration of the system with the pendulum at its lower equilibrium position. The equations for the three modes are coupled due to the righthand side in Equation (7) with the motion of the pendulum. Also, the pendulum motion is nonlinearly coupled only to the response of the first and the third translational modes; the second mode being uncoupled from the motion of the pendulum, as well as from motions of the first and the third translational modes.

As already stated, the interest in this study is on steady-state periodic solutions and their domains of attractions resulting from the excitation acting on the bottom block. For small amplitude oscillations near the third (highest) natural frequency of the 3-DOF system, it can be shown that the response of the system can be well approximated by assuming negligible contributions to the third mode and the pendulum motions from the first (rigid body) translational mode of the system. Such an analysis should follow standard arguments made when applying the method of averaging to weakly nonlinear resonant oscillations in systems with internal resonance [2]. Thus, it is reasonable to neglect the contribution of the first mode (rigid body mode) to the response of the third mode and the pendulum, that is, to let $y_{1} \approx 0$ in the pendulum's dynamical equation. Note that this does not suggest that these modes play no role in the full dynamic response of the system even at frequencies very close to the third modal resonance. This assumption has been further validated by comparing the later obtained solutions 
with those from numerical integration. Also, assuming small oscillations of the pendulum near $\theta=0$, we let $\sin \theta \cong \theta, \cos \theta \cong 1$. The resulting two equations of motion for $y_{3}$ and $\theta$ are with quadratic nonlinearities and are given by

$y_{3}^{\prime \prime}+2 \zeta_{3} y_{3}^{\prime}+\omega_{3}^{2} y_{3}+c_{3}\left(\theta^{\prime \prime} \theta+\theta^{2}\right)=c_{f} \cos \tau$,

$\theta^{\prime \prime}+2 \zeta_{p} \theta^{\prime}+\omega_{p}^{2} \theta+c_{p} y_{3}^{\prime \prime} \theta=0$.

Here, the coefficients in the model can be shown to be

$$
\begin{aligned}
\varsigma_{3} & =\varsigma_{1}(3+R) /\{\alpha(1+R)\}, \\
\omega_{3 n} & =\sqrt{(3+R) /\left\{\alpha^{2}(1+R)\right\}}, \\
c_{3} & =\gamma(-2)\left(R^{2}+2 R+3\right) /\left\{(3+R)\left(\sqrt{2 R^{2}+4 R+6}\right)\right\}, \\
c_{f} & =\varepsilon\left(R^{2}+2 R+3\right) /\left\{\alpha^{2}(3+R)\left(\sqrt{2 R^{2}+4 R+6}\right)\right\}, \\
\varsigma_{p} & =\varsigma_{2} \beta_{c} /(\alpha \sqrt{1+R}), \quad \omega_{p}=\beta_{c} /(\alpha \sqrt{1+R}), \\
c_{p} & =-2 /\left(\sqrt{2 R^{2}+4 R+6}\right) .
\end{aligned}
$$

\section{Local steady-state periodic solutions}

An approximate steady-state periodic solution for the reduced system is easily obtained through the method of harmonic balance. In this regard, one-term harmonic solutions were assumed for the approximate solution, which is reasonable for small amplitude harmonic excitation. See [4] for a comprehensive comparison between harmonic balance solutions and those obtained by numerical integration. Therefore, the solutions are assumed in the following form:

$$
\begin{aligned}
y_{3} & =Y_{3} \cos \left(\tau+\phi_{3}\right), \\
\theta & =B \cos \left(\tau / 2+\phi_{B}\right) .
\end{aligned}
$$

Following some trigonometric manipulations and solution of the resulting algebraic equations, explicit algebraic expressions for the amplitudes $Y_{3}$ and $B$ were obtained as a function of the system parameters. These are as follows:

$$
=\left\{\begin{array}{c}
c_{f}^{2} /\left\{\sqrt{\left(\omega_{3 n}^{2}-1\right)^{2}+\left(2 \varsigma_{3}\right)^{2}}\right\}, \text { when } B=0, \\
2 \sqrt{\left(\omega_{p}^{2}-1 / 4\right)^{2}+\left(\varsigma_{p}\right)^{2}} / c_{p}, \text { when } B \neq 0,
\end{array}\right.
$$

and the amplitude of the pendulum $B$ governed by the fourth-order polynomial

$$
B^{4}-\left(16 q / c_{3} c_{p}\right) B^{2}+64\left\{d p / c_{p}^{2}-c_{f}^{2} / 4\right\} / c_{3}^{2}=0,
$$

where

$$
\begin{aligned}
q= & \left(\omega_{3 n}^{2}-1\right)\left(\omega_{p}^{2}-1 / 4\right)-2 \varsigma_{3} \varsigma_{p} / c_{3 p}, \\
& \text { where } c_{3 p}=f(R), \\
d= & \left(\omega_{3 n}^{2}-1\right)^{2}+\left(2 \varsigma_{3}\right)^{2}, \quad p=\left(\omega_{p}^{2}-1 / 4\right)+\left(\varsigma_{p}\right)^{2} .
\end{aligned}
$$

Shown in Fig. 2 is a typical frequency response amplitude plot of the steady-state periodic solutions for the third mode and the pendulum, plotted as a function of $\alpha$. The parameters were selected to yield zero internal mistuning $\left(\omega_{3 n} / \omega_{p}=2\right), R=0.2, \beta_{c}=0.895$, small primary and secondary system (pendulum) dampings, $\zeta_{1}=0.005, \zeta_{2}=0.015$, and small forcing amplitude, $\varepsilon=0.02$. The procedure and results obtained here are in accordance with those obtained by Savran [19], who compared the performance of the autoparametric and dynamic absorbers for a similar system.

The periodic solutions in Fig. 2 are of two types: the semi-trivial solution and the nontrivial solution. The semi-trivial solution corresponds to a trivial pendulum solution, that is, it is with $B=0$ or $\theta=0$. Therefore, the semi-trivial solution, also termed the lockedpendulum solution, is the linear modal response of the third mode. In the nontrivial solution, both $Y_{3}$ and $B$ are nonzero. In the frequency interval (region A) between the points denoted as "pitchfork bifurcations," it is known that the semi-trivial solution has larger amplitude of response compared to the response amplitude of the third mode in the nontrivial solution [3, 4, 11]. However, at larger mistunings (regions B) the amplitude of the semi-trivial solution is smaller than that in the nontrivial solution. This highlights the limited bandwidth over which the pendulum acts as a vibration absorber.

Figure 2 displays some typical phenomena. As the excitation frequency is changed, there arise changes in stability in steady-state solution branches either through a pitchfork bifurcation or through a saddlenode bifurcation (turning point). These results are mentioned only for completeness, and typical results for this class of systems, based on averaged equations, can be found in [4] as well as in [12]. For harmonic balance 
Fig. 2 Amplitude responses for the third mode and the pendulum as a function of the normalized frequency of excitation, for a nominal set of parameters. $R=0.2$, $\beta_{c}=0.895, \zeta_{1}=0.005$, $\zeta_{2}=0.015, \varepsilon=0.02$
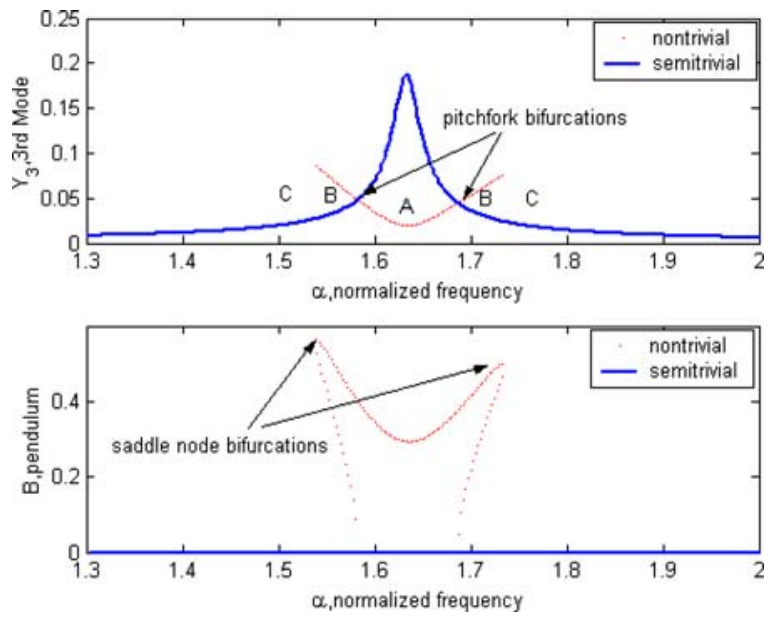

compared to the other for different initial conditions. Note that contrary to steady-state behavior, the analysis here may not be reduced to two second-order differential equations (Equations (8) and (9)) for the third locked-pendulum mode and the pendulum. So, even if the periodic solutions may essentially be confined to the four-dimensional subspace of $\left(y_{3}, y_{3}^{\prime}, \theta, \theta^{\prime}\right)$ and thus well approximated by the harmonic balance procedure outlined above, the transient motions may not be limited to only this subspace.

It is known that the boundaries of domains of attraction of attractive invariant sets in a finite-dimensional dynamical system are characterized by the stable manifolds of various hyperbolic structures, such as unstable fixed points, unstable periodic orbits, and other higher complexity solutions. Finding domains of attraction of steady-state solutions of forced nonlinear systems is a very difficult problem and not much literature exists on the subject. Tondl and colleagues have over the years (e.g., see [21]) used a method, which involves applying a disturbance over a period and observing the subsequent motion. However, such a technique, though insightful, may not necessarily capture the true domains of attraction for a system. Another noteworthy study is that of Lee and Hsu [13], who considered a harmonically excited 2-DOF planar spring-pendulum system, and used several techniques such as the cell-tocell mapping and interpolated mapping to construct the domains of attraction of stable periodic solutions. The first technique partitions the state-space into cells and the dynamics of the system is approximated by mappings over a period of forcing. Iterates of the mapping then correspond to evolution of the system in time. Escape of solutions from the cells then gives a measure 
of the domains of attraction. The interpolated mapping method finds an image corresponding to the discrete state-space model using an interpolation-based procedure. We now give a short review of the finite-time Lyapunov exponents approach to approximating domains of attraction.

\subsection{Maximal Lyapunov exponent algorithm}

Consider an $n$-dimensional dynamical system:

$\{\dot{x}(t)\}=\{f(\{x\}, t)\}$.

Let $\left\{x\left(\left\{x_{0}\right\}, t\right)\right\}$ denote the solution to Equation (14) starting at an initial condition $\left\{x_{0}\right\}$. Infinitesimal perturbations $\{v(t)\}$ about a specified trajectory $\{\bar{x}(t)\}$ satisfy the variational equation

$\{\dot{v}(t)\}=\frac{\partial\{f(\{\bar{x}\}, t)\}}{\partial\{x\}}\{v(t)\}$.

These perturbations admit the unique solution

$\{v(t)\}=\frac{\partial\left\{x\left(\left\{x_{0}\right\}, t\right)\right\}}{\partial\left\{x_{0}\right\}}\left\{v\left(t_{0}\right)\right\}$,

where $\partial\left\{x\left(\left\{x_{0}\right\}, t\right)\right\} / \partial\left\{x_{0}\right\}$ is the differential transition operator (or state transition matrix for the linear system (15)). By taking norms in Equation (16) and dividing by the perturbation $\left\|\left\{v\left(t_{0}\right)\right\}\right\| \neq 0$, the maximal stretch in the flow field at each time instant can be written as

$$
\begin{aligned}
\sup \frac{\|\{v(t)\}\|}{\left\|\left\{v\left(t_{0}\right)\right\}\right\|} & =\sup \frac{\left\|\frac{\partial\{x\}}{\partial\left\{x_{0}\right\}}\left\{v\left(t_{0}\right)\right\}\right\|}{\left\|\left\{v\left(t_{0}\right)\right\}\right\|} \\
& =\bar{\sigma}\left(\partial\{x\} / \partial\left\{x_{0}\right\}\right),
\end{aligned}
$$

where $\|\{\cdot\}\|$ is the Euclidean norm and $\bar{\sigma}(\cdot)$ is the maximum singular value of a matrix. Then, the direct (finitetime) Lyapunov exponent $[17,18]$ at a trajectory starting at $\left\{x_{0}\right\}$ is defined as

$\delta\left(\left\{x_{0}\right\}, t\right)=\frac{\ln \bar{\sigma}\left(\partial\{x\} / \partial\left\{x_{0}\right\}\right)}{\left(t-t_{0}\right)}$,

where $\bar{\sigma}\left(\partial\{x\} / \partial\left\{x_{0}\right\}\right)$ represents the maximum stretch in the flow field. Indeed, $\bar{\sigma}\left(\partial\{x\} / \partial\left\{x_{0}\right\}\right)>1$ for a region of stretch and conversely for contraction regions, whereas $\bar{\sigma}\left(\partial\{x\} / \partial\left\{x_{0}\right\}\right)=1$ represents no stretch or contraction. The aforementioned scenarios correspond to positive, negative, and zero values for $\delta\left(\left\{x_{0}\right\}, t\right)$. Thus, a positive Lyapunov exponent represents a region in state-space where trajectories are separating exponentially. In this discussion, properties of the Lyapunov exponent and the corresponding system behavior may be either over a time interval $t \in\left[t_{1}, t_{2}\right]$ or uniformly in time $\forall t>t_{0}$. This notion of having a positive Lyapunov exponent has been commonly used as a measure of chaos in dynamical systems [15, 20]. However, information about the sensitivity of system's solutions to initial conditions can be used to deduce many other convergence characteristics.

The algorithm to be used here involves solving for the finite-time Lyapunov exponent over a grid of initial conditions for a large enough time such that all solutions settle to their final states. Therefore, the longer the solution time is, the more refined the picture will become as nearby trajectories would separate (or come closer) as the time traces of their corresponding Lyapunov exponents would separate (or merge). The value of the maximal Lyapunov exponent, a measure of the sensitivity of a system to initial conditions, is expected to be maximal on the boundaries between different domains of attraction. This is evident as these boundaries exhibit the largest sensitivity to initial conditions for the system.

For the full eight-dimensional state-space of the system under study, the domains of attraction in the frequency range of interest are viewed through the separation between two physically different solutions both on the stable manifold. Geometrically, this needs to be realized in a seven-dimensional phase space. However, since visualizing such an object even via taking slices or projections into lower dimensional subspaces will not necessarily yield much insight, we opt to focus on observing the effect this object has on the domains of attraction in the $\left(\theta_{0}, \dot{\theta}_{0}\right)$ plane. In this regard, the pendulum solutions would be either a fixed-point solution at the origin, which corresponds to the semi-trivial solution for the system, or a periodic orbit (limit cycle) that corresponds to nontrivial solutions. These may be sufficient to capture the targeted behavior of the system.

Observe that the problem of finding the finite-time Lyapunov exponent is, in essence, that of finding the operator norm or largest singular value of the differential transition operator (Equation (17)). Due to the fixing of initial conditions for the six translational states to zero, the problem reduces to that of finding the largest singular value of an $8 \times 2$ matrix. It is not difficult to 
show that this reduces to finding the largest eigenvalue of a $2 \times 2$ matrix, since the remaining six singular values are zero. This means that this problem is mathematically equivalent to finding the finite-time invariant manifolds in a 2D flow field. The algorithm here uses a fourth-order fixed step Runge-Kutta approximation to the solution of Equations (1)-(4), which translates to a global truncation error of order $h^{4}$, where $h$ is the step size for time-integration. Considering $m$ different initial conditions for which the solution is to be sampled along each of the two axes yields $m^{2}$ combinations. For this eighth-order state-space model that translates to solving $8 \mathrm{~m}^{2}$ first-order differential equations. In addition, these equations need to be solved for long enough time such that steady-state behavior is reached.

\subsection{Simulation results}

The final algorithm used to produce the results presented in the following uses 100 initial condition samples $(m)$ on each axis, a step size of $0.01 \mathrm{~s}(\approx T / 40)$ where $T$ is the period of excitation, and 15,000 time steps in integration. These parameters were selected on the basis of experience from numerically obtained solutions. The domains of attraction at an excitation frequency $\omega=14.7 \mathrm{rad} / \mathrm{s}$, which corresponds to $5 \%$ external mistuning from exact resonance condition $\left(\omega_{3}=1\right)$, are shown in Fig. 3. The response curves in Fig. 2 had indicated that the semi-trivial state and a nontrivial periodic solution are stable for this excitation frequency. The data in Fig. 3a was generated via direct numerical integration for a limited range of initial condition vector $\left(\theta_{0}, \dot{\theta}_{0}\right)$ near the lower pendulum equilibrium and using a relatively coarse grid. The points "." indicate initial conditions that converge to the semi-trivial solution and the points "**" represent initial conditions that converge to the nontrivial periodic response. Figure $3 \mathrm{~b}$ is a plot of the level sets of the largest Lyapunov exponent for the same set of parameters and excitation frequency. It is evident that the two techniques agree, up to the limited discretization of the $\left(\theta_{0}, \dot{\theta}_{0}\right)$ plane used in direct numerical integration. Knowing that at $\left(\theta_{0}=0, \dot{\theta}_{0}=0\right)$, the solution is the semi-trivial one and that the boundaries between different domains correspond to the largest Lyapunov exponent, the picture becomes clear. As predicted by numerical integration, the trajectories for initial conditions near the origin converge to the fixed-point solution (semi-trivial periodic solution). This corresponds

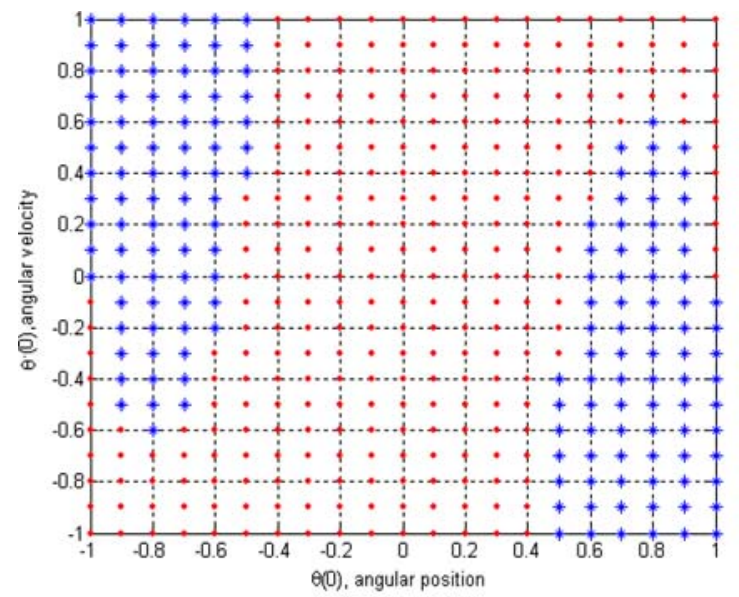

(a)

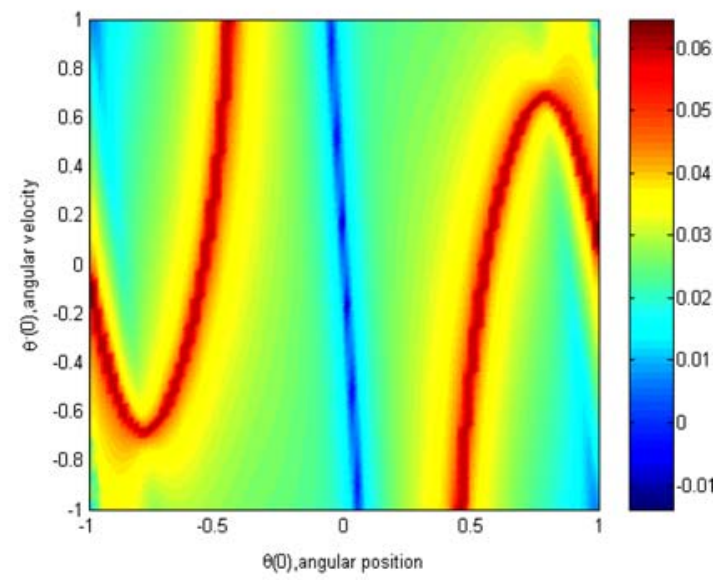

(b)

Fig. 3 Domains of attraction in the $\left(\theta_{0}, \dot{\theta}_{0}\right)$ plane at $\omega=$ $14.7 \mathrm{rad} / \mathrm{s}$. (a) Result of direct numerical integration; “.” indicate initial conditions that converged to the semi-trivial solution and "**" represent initial conditions that converged to nontrivial response. (b) Level curves of the maximal Lyapunov exponent. $R=0.2, \beta_{c}=0.895, \zeta_{1}=0.005, \zeta_{2}=0.015, \varepsilon=0.02$

to a small Lyapunov exponent, that is, strongly attractive solution. As the initial angle increases the system is more likely to be attracted to the nontrivial stable periodic orbit. This is signified by the blue region which is that of minimal stretch in the flow field or with strongest attraction for the fixed-point semi-trivial solution.

The plot also reveals variation in the rate of attraction from different initial conditions, that is, the degree of attraction within a domain. The simulation results starting with different initial conditions (Fig. 4) show that the regions with different degrees of attraction could correspond to quite different global system behavior. The three cases in Fig. $4 \mathrm{a}-\mathrm{c}$ lie within the central 

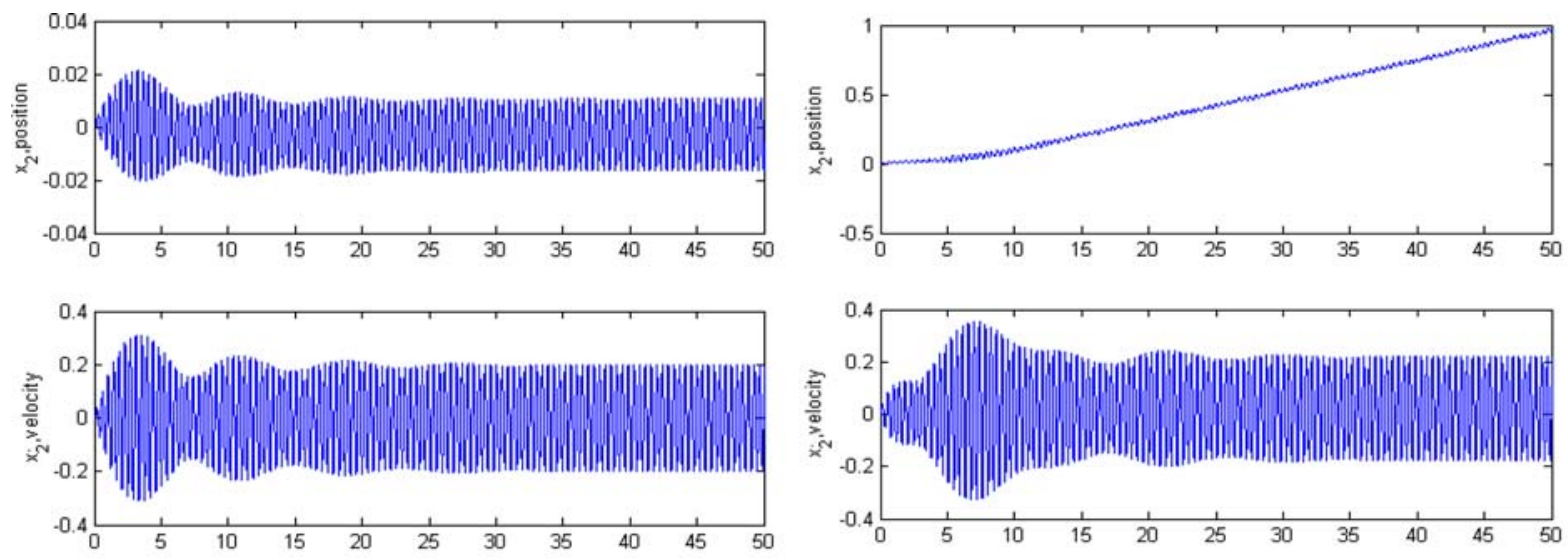

(a)

(b)
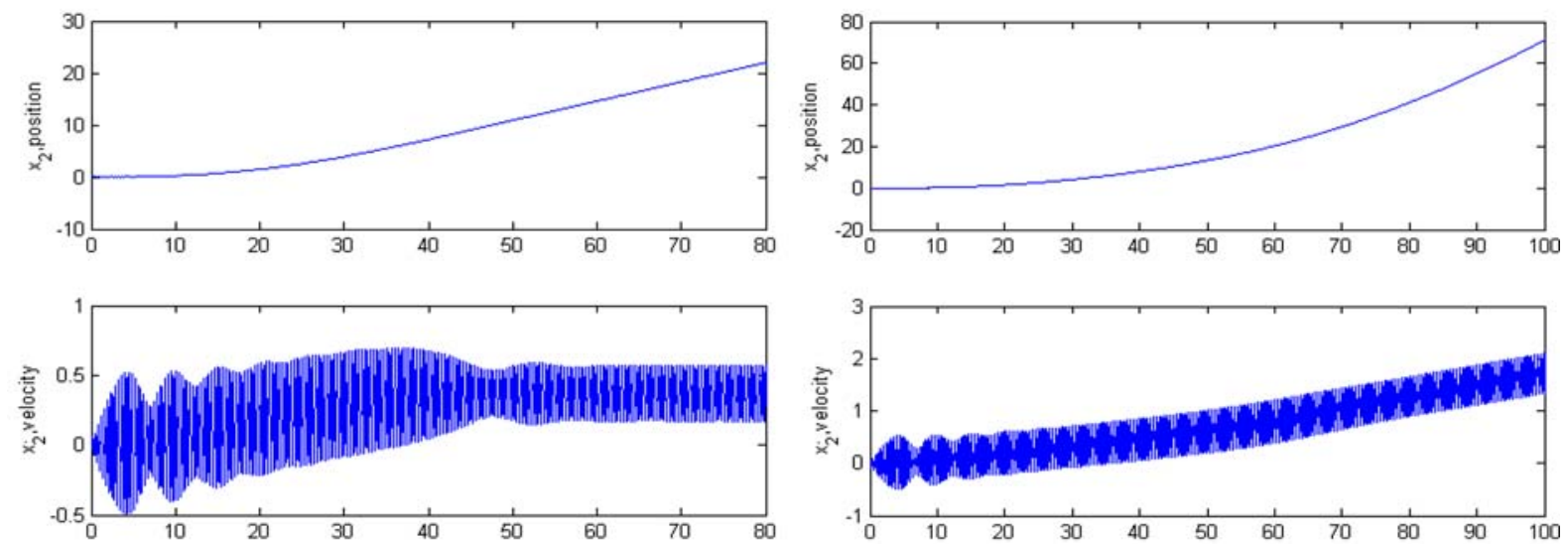

(c)

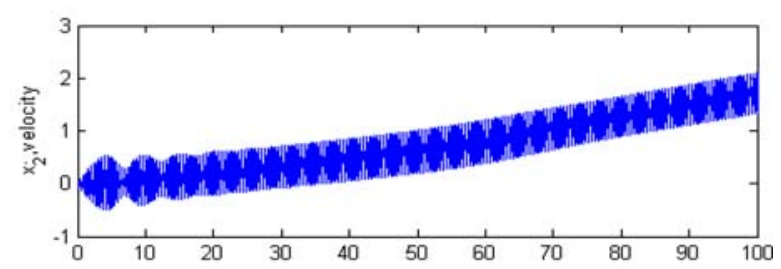

(d)

Fig. 4 Time response (position $x_{2}$ and velocity $\dot{x}_{2}$ ) of second mass for different initial conditions in $\left(\theta_{0}, \dot{\theta}_{0}\right)$ plane for the case in Fig. 3. (a) $\left(\theta_{0}=0, \dot{\theta}_{0}=1\right)$, (b) $\left(\theta_{0}=0.2, \dot{\theta}_{0}=1\right)$, (c) $\left(\theta_{0}=0.5\right.$,

regions of Lyapunov exponent $\delta,(-0.01<\delta<0.01)$, $(0.01<\delta<0.03)$, and $(0.03<\delta<0.04)$ and correspond to three different semi-trivial solutions based on different contributions from the rigid body mode. Observe also that the responses for the cases in Fig. $4 \mathrm{c}$ and $\mathrm{d}$, which correspond to initial conditions on either side of the boundary between different domains, have very similar time traces up to a critical time beyond which solutions' paths deviate. It is noteworthy that for a very small change in initial conditions, the steady-state pendulum oscillations shift from an amplitude of 0 to about $0.5 \mathrm{rad}$. Note that the results here in Fig. 5 were plotted for $x_{2}$ and $x_{2}^{\prime}$ rather than $\theta$ and $\theta^{\prime}$ to show the role of coupling in the dynamics of the complete system. Furthermore, even though the drift in solutions arise for $x_{1}, x_{2}, x_{3}$, these contribute a Lyapunov exponent equal to zero. Thus, as long as there is a positive Lyapunov

$\left.\dot{\theta}_{0}=-0.3\right),(\mathrm{d})\left(\theta_{0}=0.5, \dot{\theta}_{0}=-0.4\right) . \omega=14.7 \mathrm{rad} / \mathrm{s}, R=0.2$, $\beta_{c}=0.895, \zeta_{1}=0.005, \zeta_{2}=0.015, \varepsilon=0.02$

exponent, the rigid body translations do not show up in our calculations.

The next simulation results show the effect of external mistuning. Figure 5a shows the Lyapunov exponent level curves at the same frequency and parameters of Fig. 3a but over an extended range in the pendulum's phase plane. As expected, the structure is quite complex further away from the fixed point at the origin. Figure $5 \mathrm{~b}$ shows the level curves for the excitation frequency $\omega=16.2 \mathrm{rad} / \mathrm{s}$, which also corresponds to $5 \%$ external mistuning but now above the zero mistuning point, which is at $\omega=15.45 \mathrm{rad} / \mathrm{s}$. The behavior in this case is quite distinct even in regions somewhat close to the origin. In fact, a much larger region of initial conditions converges to the fixed point at the origin, including some initial conditions much closer to the upper equilibrium position. Recall that the stable manifolds 


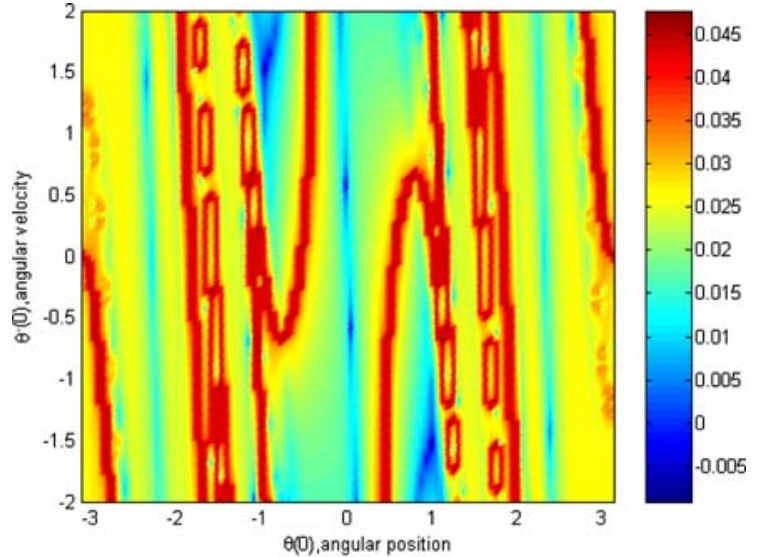

(a)

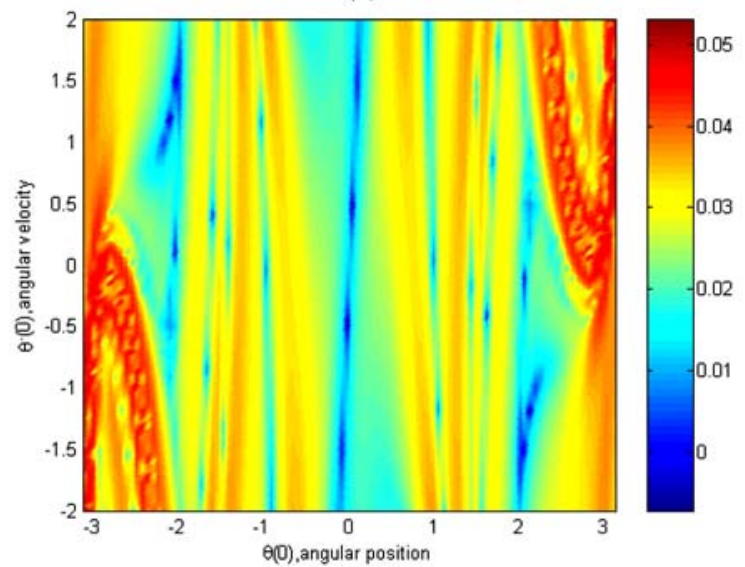

(b)

Fig. 5 Lyapunov exponent level curves in $\left(\theta_{0}, \dot{\theta}_{0}\right)$ plane. (a) $\omega=$ $14.7 \mathrm{rad} / \mathrm{s}$, (b) $\omega=16.2 \mathrm{rad} / \mathrm{s} . R=0.2, \beta_{c}=0.895, \zeta_{1}=0.005$, $\zeta_{2}=0.015, \varepsilon=0.02$

of the invariant repelling sets play a major role in shaping the domains of attraction. Reverting back to Fig. 2 , one notes the asymmetrical nature of the pendulum solutions. This asymmetry leads to the two periodic orbits being very close to each other in the phase space, for this mistuning. As a result, the unstable orbit repels most of the trajectories away from its nearby stable orbit, which then settle to the fixed point.

Next, the global behavior near bifurcation points is explored. Let us consider the two important frequencies of $\omega=14.6$ and $14.9 \mathrm{rad} / \mathrm{s}$, which are very close to a saddle-node (to its right) and a pitchfork bifurcation (to its left), respectively. The corresponding level curves for the finite-time Lyapunov exponent are shown in Fig. 6.

Observe that for $\omega=14.6 \mathrm{rad} / \mathrm{s}$, the domains of attraction picture is similar to that for $\omega=14.7 \mathrm{rad} / \mathrm{s}$,

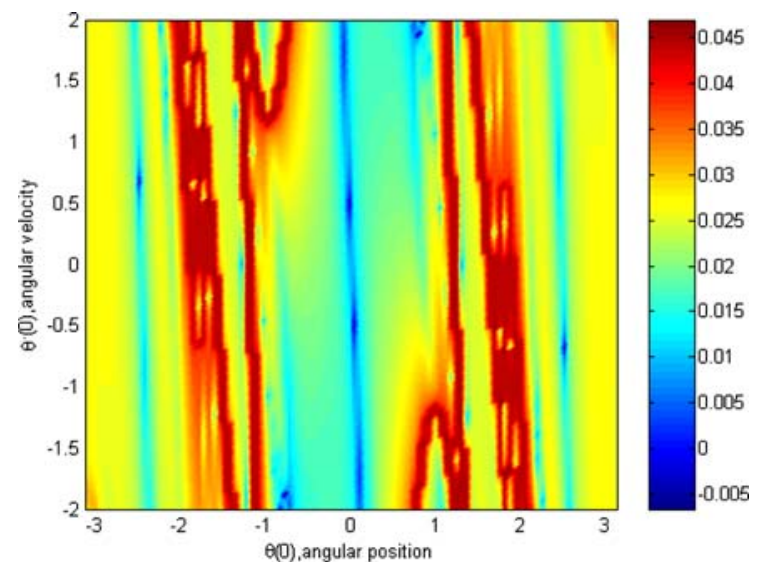

(a)

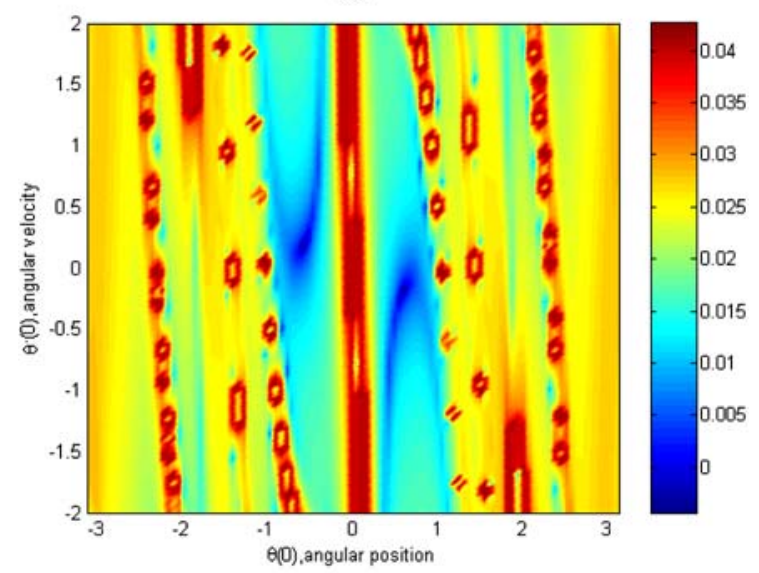

(b)

Fig. 6 Lyapunov exponent level curves in $\left(\theta_{0}, \dot{\theta}_{0}\right)$ plane. (a) $\omega=$ $14.6 \mathrm{rad} / \mathrm{s}$, (b) $\omega=14.9 \mathrm{rad} / \mathrm{s} . R=0.2, \beta_{c}=0.895, \zeta_{1}=0.005$, $\zeta_{2}=0.015, \varepsilon=0.02$

except that the regions of attraction for the semi-trivial solution are appreciably stretched in size. This is quite expected as this parameter value is closer to the saddlenode bifurcation point $(\omega=14.55 \mathrm{rad} / \mathrm{s})$ below which only the semi-trivial solution exists. This bifurcation, which corresponds to hysteretic behavior or the commonly referred to jump phenomenon, is one incentive for pursing the domains of attraction. The other significant parameter values are those in the vicinity of a pitchfork bifurcation, such as that for $\omega=14.9 \mathrm{rad} / \mathrm{s}$. Here, the semi-trivial solution is losing its stability. This is indicated by the fact that the origin in $\left(\theta_{0}, \dot{\theta}_{0}\right)$ plane itself is close to the boundary between solutions, that is, a region of weakest attraction. Here, the unstable pendulum orbit (see Fig. 2) is very small and surrounding the trivial pendulum solution. Thus, on this scale it looks like that the origin in $\left(\theta_{0}, \dot{\theta}_{0}\right)$ plane is unstable 


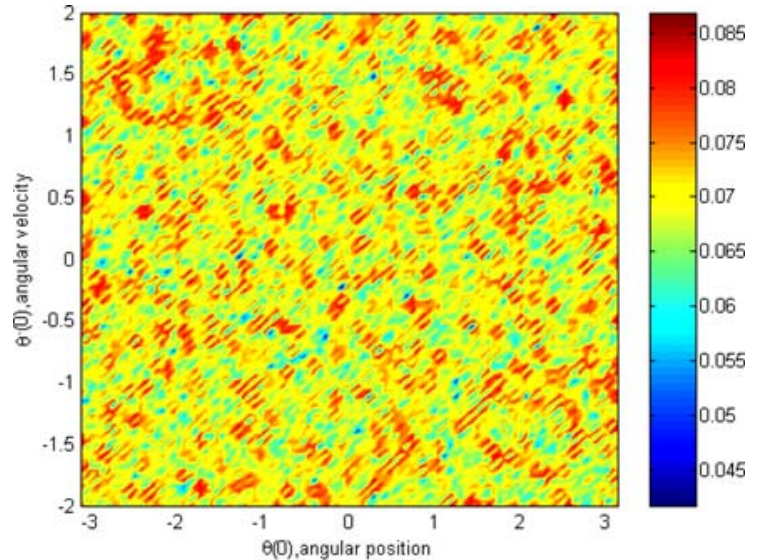

(a)

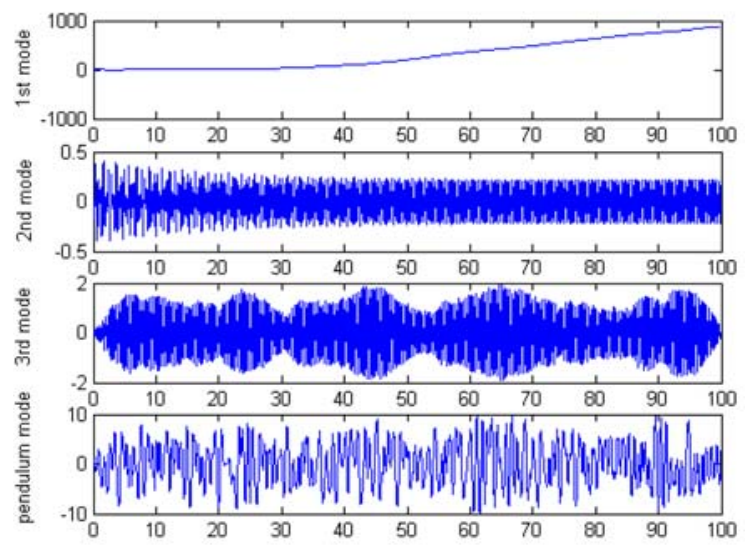

(b)

Fig. 7 Chaotic response of the system at large amplitude of excitation. (a) Level curves of the finite-time Lyapunov exponent in $\left(\theta_{0}, \dot{\theta}_{0}\right)$ plane. (b) Modal time responses with initial condition $\left(\theta_{0}=1, \dot{\theta}_{0}=0\right)$. Frequency $\omega=15.45 \mathrm{rad} / \mathrm{s}$ and amplitude $\varepsilon=$ 1 of excitation

and repelling trajectories as represented by the red area around $\theta=0$. The plot in Fig. $6 \mathrm{~b}$ then mostly consists of pairs of level curves settling onto a periodic orbit.

Finally, we illustrate the global characterization of motion of the system for sufficiently large amplitude of excitation $\varepsilon=1$ (see Fig. 7). This case corresponds to chaotic oscillations of the spring-mass-damperpendulum system. In contrast to all previous cases, the Lyapunov exponent values here are all positive over the pendulum $\left(\theta_{0}, \dot{\theta}_{0}\right)$ plane though they are not necessarily large since no significant change in the final state takes place for different trajectories. It is noteworthy that no significant changes in the structure are observed for larger excitations, e.g., when $\varepsilon=10$, and only the intensity of chaotic mixing is just increased. Another interesting observation (see Fig. 7b) here is that despite the chaotic dynamics, the response of the second mode of the system is invariably that of a linear harmonically driven system, as analytically predicted, with the excitation amplitude linearly scaling the steady-state amplitude. This is in contrast to the obviously chaotic behavior of the third mode, a result which is driven by the fact that the second mass, which is coupled to the pendulum, does not contribute to the second mode's dynamics.

\section{Conclusions}

In this work, we studied the global dynamics and domains of attraction for different periodic solutions of an autoparametric spring-mass-pendulum system. Through a modal decoupling procedure, the coupling between the pendulum and system modes was identified. In addition to the autoparametric coupling with the third flexible mode, coupling with a rigid body mode was also identified. It was shown that rigid body dynamics of the system are excited even for zero initial conditions for the rigid body mode and excitation near the third mode, which depends on the pendulum's transients. Though local steady-state weakly nonlinear behavior is well understood, the transient behavior and asymptotic convergence of solutions to a fixed point or a periodic orbit in a critical region of frequencies bounded by pitchfork and saddle-node bifurcations depends on domains of attraction. Furthermore, global and strongly nonlinear behavior is also of interest. A study of characterization of the domains of attraction was performed through the development of level curves of the finite-time Lyapunov exponent in a global region in the pendulum's plane. This approach, based on a dynamical performance index rather than on a discretization or interpolation technique, proved to be very rewarding and insightful. It provided both relative and absolute measures of global convergence to invariant sets. Furthermore, its conceptual robustness allows for global and strongly nonlinear conditions to be studied without concerns of adequacy of an iteration size or other equivalent parameter. Using local steady-state solutions as a guide, the behavior of the three coupled modes for the key cases of interest was captured including strongly excited chaotic solutions. 


\section{References}

1. Nayfeh, A.H., Balachandran, B.: Modal interactions in dynamical and structural systems. Appl. Mech. Rev. 42, 175201 (1989)

2. Nayfeh, A.H.: Nonlinear Interactions. Wiley, New York (2000)

3. Hatwal, H., Mallik, A.K., Ghosh, A.: Nonlinear vibrations of a harmonically excited autoparametric system. J. Sound Vib. 81, 153-164 (1980)

4. Bajaj, A.K., Chang, S.I., Johnson, J.: Amplitude modulated dynamics of a resonantly excited autoparametric two degreeof-freedom system. Nonlinear Dyn. 5, 433-457 (1994)

5. Cuvalci, O., Ertas, A.: Pendulum as vibration absorber for flexible structures: experiments and theory. ASME J. Vib. Acoust. 118, 558-566 (1996)

6. Cuvalci, O.: The effect of detuning parameters on the absorption region for a coupled system: a numerical and experiments study. J. Sound Vib. 229(4), 837-857 (2000)

7. Song, Y., Sato, H., Iwata, Y., Komatsuzaki, T.: The response of a dynamic vibration absorber system with a parametrically excited pendulum. J. Sound Vib. 259(4), 747-759 (2003)

8. Cartmell, M., Lawson, J.: Performance enhancement of an autoparametric vibration absorber by means of computer control. J. Sound Vib. 177, 173-195 (1994)

9. Oueini, S.S., Nayfeh, A.H., Pratt, J.R.: A review of development and implementation of an active nonlinear vibration absorber. Arch. Appl. Mech. 69, 585-620 (1999)
10. Den Hartog, J.P.: Mechanical Vibrations. McGraw Hill, New York (1956)

11. Banarjee, B., Bajaj, A.K., Davies, P.: Resonant dynamics of a chain of identical linear oscillators coupled to a nonlinear oscillator. In: Nonlinear Dyn. Controls, ASME, Vol. AMD 91, pp. 231-237 (1996)

12. Vyas, A., Bajaj, A.K.: Dynamics of autoparametric vibration absorbers using multiple pendulums. J. Sound Vib. 246, 115135 (2001)

13. Lee, W.K., Hsu, C.S.: A global analysis of a harmonically excited spring-pendulum system with internal resonance. J. Sound Vib. 171, 335-359 (1994)

14. Hsu, C.S.: Cell-To-Cell Mappings. Springer, New York (1987)

15. Moon, F.C.: Chaotic and Fractal Dynamics: An Introduction for Scientists and Engineers. Wiley, New York (1992)

16. Haller, G.: Finding finite-time invariant manifolds in twodimensional velocity fields. Chaos 10, 99-108 (2000)

17. Haller, G.: Distinguished material surfaces and coherent structures in 3D fluid flows. Physica D 149, 248-277 (2001)

18. Haller, G.: Lagrangian coherent structures from approximate velocity data. Phys. Fluids A 14, 1851-1861 (2002)

19. Savran, C.A.: A comparative study of the dynamic and pendulum absorbers. BSME Honors thesis, Purdue University, West Lafayette, IN (1998)

20. Nayfeh, A.H., Balachandran, B.: Applied Nonlinear Dynamics. Wiley, New York (1995)

21. Tondl, A., Ruijgorg, T., Verhulst, F., Nabergoj, R.: Autoparametric Resonance in Mechanical Systems. Cambridge University Press, Cambridge, UK (2000) 